\title{
TRACE observations of driven loop oscillations
}

\author{
I. Ballai ${ }^{1}$, D. B. Jess ${ }^{2}$, and M. Douglas ${ }^{1}$ \\ ${ }^{1}$ Solar Physics and Space Plasma Research Centre (SP2 RC), Department of Applied Mathematics, The University of Sheffield, \\ Sheffield, S3 7RH, UK \\ e-mail: [i.ballai; mark.douglas]@sheffield.ac.uk \\ 2 Astrophysics Research Centre, School of Mathematics and Physics, Queen's University, Belfast, BT7 1NN, Northern Ireland, UK \\ e-mail: d.jess@qub.ac.uk
}

Received 16 February 2010 / Accepted 21 July 2011

\section{ABSTRACT}

\begin{abstract}
Aims. On 13 June 1998, the TRACE satellite was fortuitously well placed to observe the effects of a flare-induced EIT wave in the corona, and its subsequent interaction with coronal magnetic loops. In this study, we use these TRACE observations to corroborate previous theoretical work, which determined the response of a coronal loop to a harmonic driver in the context of ideal magnetohydrodynamics, as well as estimate the magnetic field strength and the degree of longitudinal inhomogeneity.

Methods. Loop edges are tracked, both spatially and temporally, using wavelet modulus maxima algorithms, with corresponding loop displacements from its quiescent state analysed by fitting scaled sinusoidal functions. The physical parameters of the coronal loop are subsequently determined using seismological techniques.

Results. The studied coronal loop is found to oscillate with two distinct periods, $501 \pm 5 \mathrm{~s}$ and $274 \pm 7 \mathrm{~s}$, which could be interpreted as belonging to the fundamental kink mode and first harmonic, or could reflect the stage of an overdriven loop. Additional scenarios for explaining the two periods are listed, each resulting in a different value of the magnetic field and the intrinsic and sub-resolution properties of the coronal loop. When assuming the periods belong to the fundamental kink mode and its first harmonic, we obtain a magnetic field strength inside the oscillating coronal loop of $2.0 \pm 0.7 \mathrm{G}$. In contrast, interpreting the oscillations as a combination of the loop's natural kink frequency and a harmonic EIT wave provides a magnetic field strength of $5.8 \pm 1.5 \mathrm{G}$. Using the ratio of the two periods, we find that the gravitational scale height in the loop is $73 \pm 3 \mathrm{Mm}$.

Conclusions. We show that the observation of two distinct periods in a coronal loop does not necessarily lead to a unique conclusion. Multiple plausible scenarios exist, suggesting that both the derived strength of the magnetic field and the sub-resolution properties of the coronal loop depend entirely on which interpretation is chosen. The interpretation of the observations in terms of a combination of the natural kink mode of the coronal loop, driven by a harmonic EIT wave seems to result in values of the magnetic field consistent with previous findings. Other interpretations, which are realistic, such as kink fundamental mode/first harmonic and the oscillations of two sub-resolution threads result in magnetic field strengths that are below the average values found before.
\end{abstract}

Key words. magnetohydrodynamics (MHD) - Sun: corona - magnetic fields - Sun: oscillations

\section{Introduction}

Sudden energy releases in the solar atmosphere are known to generate large-scale global waves propagating over long distances. The energy stored in these waves can be released by traditional dissipative mechanisms, but might also be transferred to magnetic structures that come into contact with global waves. In this context, EIT waves generated by coronal mass ejections (CMEs) and/or flares could interact with coronal loops, resulting in the generation of kink modes, i.e. oscillations that experience periodic movement about the loop's symmetry axis. These generated loop oscillations have been used as a basic ingredient in one of solar physics most dynamically expanding fields, namely coronal seismology. Depending on the orientation of the studied wave, coronal seismology can be further divided into local (Roberts et al. 1984; Nakariakov et al. 1999; Banerjee et al. 2007; Verth et al. 2007) and global seismology (Ballai et al. 2005; Ballai 2007). Although local and global seismology appear to be two different aspects of coronal physics, in reality they are closely related.

Coronal seismology is based on theoretical relations (called dispersion relations) linking plasma parameters, such as the plasma density, to wave parameters, such as the wave frequency, in a precise way. In general, plasma parameters are determined from wave parameters, which themselves are determined observationally. The dispersion relations for many simple (and some quite complicated) plasma structures under the assumptions of ideal magnetohydrodynamics (MHD) are well known; they were derived long before accurate EUV observations were available (Edwin \& Roberts 1983; Roberts et al. 1984) using simplified models within the framework of ideal and linear MHD. Although the accurate interpretation of many observations can be difficult because of the insufficient spatial and temporal resolutions of present satellites, considerable amounts of information about the state of the plasma, and the structure and magnitude of the coronal magnetic field, can still be obtained.

Most of the previous studies of waves and oscillations in coronal loops have focussed on the (local) oscillation itself, without considering the more global cause of the oscillation. One of the first studies where the nature of a local loop oscillation was investigated in terms of the type of driver (global EIT waves) was the study by Ballai et al. (2008). These authors analysed the pattern of possible oscillations (treated as an initial value problem) that can be recovered in a simplified loop model under the influence of, e.g. harmonic drivers interacting with loops. Their main conclusion, which is used in the present study, was that for 


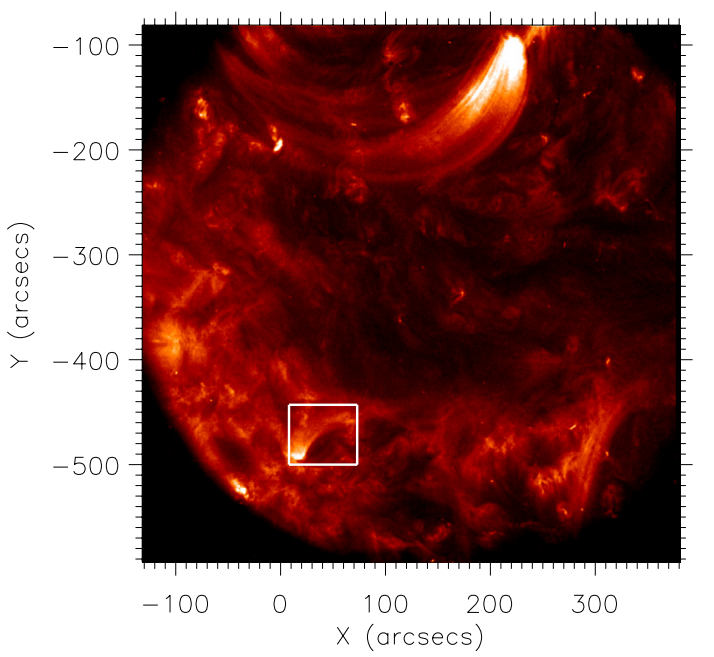

Fig. 1. TRACE field-of-view at $171 \AA$ immediately prior to the arrival of the EIT wave front. The white box indicates the coronal loop under investigation in the present study. Axes are in solar heliocentric arcseconds.

a certain range of driver periods, the loop supports oscillations whose period is a superposition of the driver's period and the natural period of the loop. A similar analysis of a 2D loop was carried out by Selwa et al. (2010).

The aim of this paper is to investigate, using TRACE EUV observations, the characteristics of a coronal loop that oscillates under the influence of an external driver. The special circumstances of this event reside in the possibility of connecting the loop oscillation to a global EIT wave, presenting the phenomena of loop oscillations in a much wider context.

\section{Observations and data analysis}

In contrast to the SOHO/EIT instrument, the TRACE/EUV imager has good temporal and spatial resolution, suitable for observing waves in the MHD domain. However, the field-of-view of TRACE is merely $8.5 \mathrm{~min}$ of arc, compared to SOHO/EIT's full disc images of $45 \mathrm{~min}$ of arc. Thus, obtaining TRACE data close to the source of an EIT wave requires a small measure of good fortune; one such instance occurred on 13 June 1998. On this day, a coronal wave was initiated just south of the field of view of TRACE at approximately 15:23 UT by a GOES C2.9 flare. The maximum flare intensity occurred between the eastern part of the main negative polarity and the western part of the main positive polarity regions of AR 8237 (S25W04 in the solar heliographic coordinate system) at 15:33 UT, where a filament was also observed in $\mathrm{H} \alpha$. Furthermore, this event was associated with a halo CME observed in white light by LASCO C2 with a leading edge moving at $190 \mathrm{~km} \mathrm{~s}^{-1}$ (Delannée 2000). A limited study of this event has already been carried out by Wills-Davey \& Thompson (1999), with the authors interpreting the disturbance as a fast magnetoacoustic wave.

TRACE images in the $171 \AA$ and $195 \AA$ bandpasses acquired during 15:00-19:00 UT show the progress of the wave (activity over an extended area of the field-of-view), and from the SOHO/EIT instrument a concomitant EIT wave is visible (Wills-Davey \& Thompson 1999). The TRACE data also reveal how the EIT wave front causes the positioning of some coronal loops to be disturbed. The particular loop under investigation is situated in the south-eastern part of Fig. 1, with a zoom into the region of interest in Fig. 2.
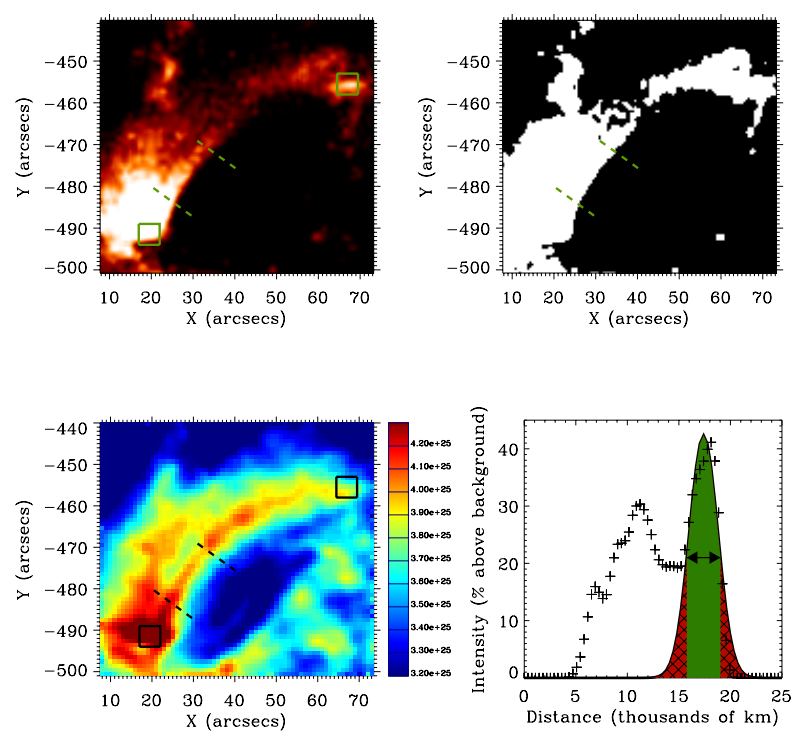

Fig. 2. Zoomed images of the TRACE field-of-view contained within the white box in Fig. 1. The upper-left image displays a true intensity image, while the upper-right panel reveals the corresponding loopedge binary map used in the tracking algorithm of Jess et al. (2008). Locations of the loop footpoints are highlighted with green boxes in the left panel, giving an approximate loop length, $L=72.9 \pm 2.8 \mathrm{Mm}$. An $11000 \mathrm{~km}$ segment, incorporating a brightly emitting section of the loop edge, which is used in the search for higher harmonics is contained within the green dashed lines plotted in the upper panels. The lower-left panel displays an emission measure map, derived from near-simultaneous $171 \AA$ and $195 \AA$ TRACE images, with the corresponding colour scale in units of $\mathrm{cm}^{-5}$. Black boxes and dashed lines represent characteristics identical to those described above for the upper-left panel. The loop arcade intensity (+), averaged between the green dashed lines in the upper-left panel, is plotted in the lower-right panel as a percentage above the quiescent background. A Gaussian fit to the coronal loop under investigation is displayed using a solid black line. Green shading represents loop intensities greater than the fitted half-maximum, while cross-hatched red shading indicates regions of the loop structure with intensities below the half-maximum. Arrows mark the full-width at half-maximum, chosen to represent an estimate of the loop depth, found here to be $\approx 8$ pixels, or $\approx 3000 \mathrm{~km}$.

Using the standard calibration/correction packages available in the SolarSoft library, the TRACE data was processed in the usual way: spikes from cosmic ray hits were removed, data frames were normalized to a constant exposure time, and the image sequence was de-rotated. The TRACE satellite entered the Southern Atlantic Anomaly at approximately 15:50 UT, and images up to this point clearly display a propagating wave front.

\subsection{Temporal analysis of loop edge}

The characteristics of a global EIT wave were studied in detail by Ballai et al. (2005), who demonstrated that the global disturbance is a wave with an average periodicity of approximately $400 \mathrm{~s}$. To accurately probe the effects of this wave front on more rigidly fixed coronal loop structures, the loop edge was tracked spatially and temporally using a wavelet modulus maxima technique described by Jess et al. (2008). Since the $195 \AA$ response function is somewhat lower than that of the $171 \AA$ passband, longer exposure times are required to provide the same signal as the $171 \AA$ channel (a $41 \mathrm{~s}$ average exposure time at $195 \AA$, versus $24 \mathrm{~s}$ at $171 \AA$ images). As a result, rapidly moving structures may be blurred in the $195 \AA$ passband because of their substantially 
longer exposure times. Furthermore, the $171 \AA$ images are at full resolution $\left(1\right.$ pixel $\left.=0.499^{\prime \prime}\right)$, whereas the $195 \AA$ data are $2 \times 2$ binned, resulting in a pixel size of $0.998^{\prime \prime}$. Through use of blind iterative deconvolution techniques, Golub et al. (1999) and Lin et al. (2001) determined the absolute spatial resolution of the TRACE instrument to be $1.25^{\prime \prime}$. Since the $171 \AA$ data is not degraded by spatial binning, it enables us to monitor small-scale disturbances more clearly in coronal loop structures. As a result, only images taken in the $171 \AA$ bandpass could be tracked with an average cadence of $90 \mathrm{~s}$. First, a segment of the loop edge, between the loop footpoint and apex, was passed through a Laplacian filter to sharpen the images and make the edge of the structure more pronounced. Next, a lower intensity threshold was applied to the data to remove contributions from the underlying quiet Sun. A threshold of the background mean flux plus $4.5 \sigma$ was used, which produces the image displayed in the upper-left panel of Fig. 2. To emphasize feature edges and remove shallow intensity gradients, we used a binary format for the feature mapping. All pixels of values above the lower flux intensity threshold defined above were assigned a value of " 1 ". Those pixels which lie below the threshold are assigned a value of " 0 ", producing the binary map shown in the upper-right panel of Fig. 2. To assist the analysis, each image was rotated by $45^{\circ}$ clockwise until the southern edge of the loop structure was parallel with the $x$-axis. This caused loop displacements to be confined to the direction of the $y$-axis, as a function of the loop length ( $x$-axis). TRACE images acquired between the initiation of oscillatory behaviour at 15:29 UT, and 15:50 UT when the instrument entered the Southern Atlantic Anomoly, were binary mapped for edge detection and tracking using the methodology described by Jess et al. (2008).

Utilizing the resulting loop-edge positions, the displacement of the loop segment as a function of time from its quiescent state was established for 30 pixels $(\approx 11000 \mathrm{~km}$; see dashed lines in Fig. 2) along the loop edge between the footpoint and apex. Owing to the minimal shifts in the loop equilibrium position with time, and the need to minimise the number of free fitting parameters, no long-term trends were subtracted from the original time series. The loop-edge displacement curve was then fitted using sinusoids of varying frequency and amplitude. The best fit sinusoid was then subtracted from the original data to produce a residual time series. The residual data, again, was modelled with the best-fit sinusoid function. To determine the success of the fitting process, Kolmogorov-Smirnov and crosscorrelation statistics (Press et al. 1992; Christian et al. 1998; Jess et al. 2007a,b) were determined for each stage of the sinusoid fitting process, as well as for the combined fit with respect to the original time series. An example of this process is displayed in Fig. 3, where Kolmogorov-Smirnov statistics for the 501 s, 274 s, and combined fits are $88 \%, 91 \%$, and $92 \%$, respectively. Crosscorrelation statistics for the same fitting functions are $90 \%, 74 \%$, and $98 \%$, respectively, indicating that these sinusoid functions recreate the observed data with a high degree of precision.

To validate this result, the process of loop isolation, tracking, and sinusoid-fitting was repeated for image sequences that had been spatially degraded using $2 \times 2,3 \times 3,5 \times 5$, and $7 \times 7$ binning intervals. While spatial binning has no effect on a robust periodic signature, it will help us to reduce any artefacts present in the data, such as those caused by detector noise and/or cosmic ray hits. These independent analyses yielded consistent results, for which periodicities overlapped with those established above, implying that the oscillation occurs over a large spatial scale, and is unaffected by varying smoothing intervals. As described
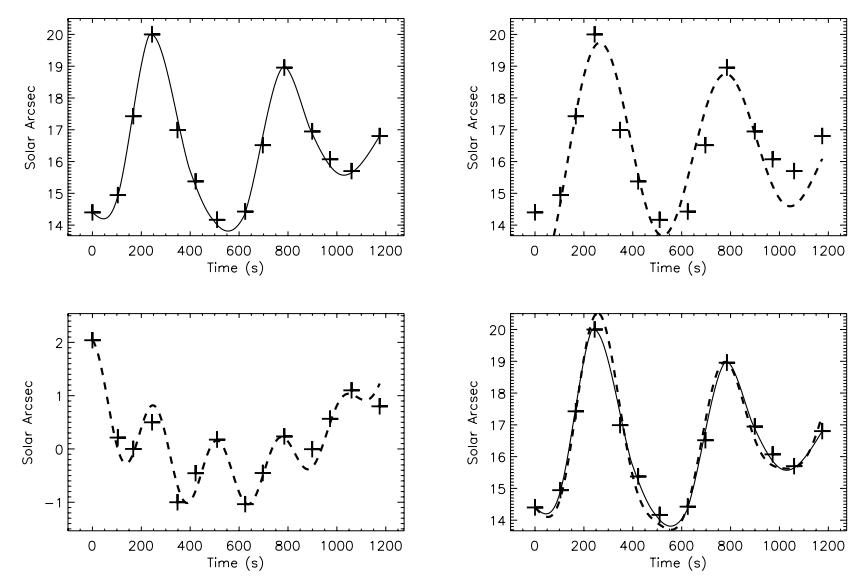

Fig. 3. Top left: a typical transverse displacement of one pixel along the loop segment $(+)$, joined together using a spline fit to assist visualisation of the motion path (solid line). Top right: the original transverse displacement $(+)$, overplotted with a $501 \mathrm{~s}$ period sinusoidal signal (dashed line). Bottom left: the residual (501 s period sinusoid subtracted from the original motion path) transverse displacement $(+)$, overplotted with a $274 \mathrm{~s}$ period sinusoidal signal (dashed line). Bottom right: the original data points $(+)$ and spline fit (solid line) shown in the upper-left panel, overplotted with the combined $501 / 274$ s period sinusoidal fits. These combined fits, with respect to the original time series, result in Kolmogorov-Smirnov and cross-correlation statistics of $92 \%$ and $98 \%$, respectively, indicating a high degree of accuracy.

in Van Doorsselaere et al. (2007), repeating this procedure for, and averaging over, many loop positions and binning intervals (30 loop positions $\times 5$ binning values $=150$ independent measurements in this instance) allows the associated errors to be drastically reduced. Following a similar methodology, we derive best-fit periodicities of $501 \pm 5 \mathrm{~s}$ and $274 \pm 7 \mathrm{~s}$, both of which are above the limiting Nyquist period $(\approx 180 \mathrm{~s})$ of the dataset.

\subsection{Properties of the coronal loop}

The fact that two different periods have been recovered from the same structure allows us to uncover properties of the loop that cannot be directly measured. We use a simple semicircular model for the loop, where the loop length, $L$, is given by $\pi D / 2$, where $D$ is the distance between footpoints. As the TRACE field of view is nearly at the solar disc centre, and the loop does not appear to be tilted at a significant angle, this model is likely to be accurate. The distance $D$ is measured to be $128 \pm 5$ TRACE pixels, corresponding to an absolute diameter of $D=46.4 \pm 1.8 \mathrm{Mm}$. Thus, we are dealing with a relatively short loop, with a corresponding estimated loop length of $L=72.9 \pm 2.8 \mathrm{Mm}$.

The electron number density of the loop (and its environment) can be estimated using a differential emission measure technique by comparing the intensities of the loop detected at different wavelengths. This filter-ratio method is based on the assumption that a structure seen in both $171 \AA$ and $195 \AA$ bandpasses will be isothermal, with an associated temperature falling between the two peak temperatures of the TRACE filter response functions (Aschwanden et al. 2000). Using nearsimultaneous $171 \AA$ and $195 \AA$ TRACE images, and following the methodology described in Aschwanden (2004), we calculate the emission measure, $E M$, as $E M=(4.0 \pm 0.2) \times 10^{25} \mathrm{~cm}^{-5}$, averaged over the 30-pixel loop segment used in Sect. 2.1 (lower-left panel of Fig. 2). This value represents the emission 
integrated over the entire line-of-sight. The relationship between emission measure and electron number density, $n_{\mathrm{e}}$, in a source plasma structure of depth, $d$, is given by

$n_{\mathrm{e}}(x, y)=\sqrt{\frac{E M(x, y)}{d}}$,

where $x$ and $y$ are the spatial coordinates (Aschwanden 2004). Assuming a cylindrical loop structure, and using the full-width at half maximum to estimate the loop cross-section, we estimate the loop depth to be $8 \pm 1$ pixels, or $(29.2 \pm 3.6) \times 10^{7} \mathrm{~cm}$ (lowerright panel of Fig. 2). This allows us to calculate the electron number density in the loop as $n_{\mathrm{e}}=(3.7 \pm 2.3) \times 10^{8} \mathrm{~cm}^{-3}$. Using a quiescent plasma electron number density (the region outside the loop) of $1 \pm 0.5 \times 10^{8} \mathrm{~cm}^{-3}$ in line with previous estimates (see, e.g. Fludra et al. 1999; Ugarte-Urra et al. 2002), we can determine the loop filling factor to be $3.7 \pm 0.4$. For brightly emitting coronal loops, it can be assumed that all of the emission measure comes directly from the loop itself. However, for relatively dim loops, similar to the one under investigation here, a significant portion of the emission will come from plasma in the immediate vicinity of the loop. As a result, the derived electron number densities will represent the upper boundaries of actual values.

\subsection{Application of coronal seismology}

Coronal seismology, initiated after high-resolution observations of waves and oscillations in coronal structures became available, can provide relatively accurate estimates of quantities that cannot be measured directly or are below the instrument's resolution limit. Using a combination of observations and theoretical models, answers to the problems of the magnitudes and structuring of coronal magnetic fields, density scale-heights, etc., have since been recovered (Nakariakov et al. 1999; Andries et al. 2005, 2009; Verth et al. 2008; Ruderman \& Erdélyi 2009).

In this context, one of the most promising methods for investigating the internal structure of coronal loops is based on the $P_{1} / P_{2}$ ratio of the fundamental $\left(P_{1}\right)$ to first harmonic $\left(P_{2}\right)$ modes of an oscillation. In a homogeneous, non-stratified atmosphere the period ratio is 2 . However, longitudinal variations in the properties of the plasma (i.e. variations along the loop) can make this ratio to deviate either below or above 2 . It is believed that magnetic effects produce a ratio that is greater than 2 because of the magnetic divergence caused by changes in the crosssectional area of the loop (De Moortel \& Brady 2007; O'Shea et al. 2007). In this case, the magnetic effect on the period of oscillation dominates any other effects, such as density stratification. In contrast, if the ratio of the two periods is less than 2 , then the change is attributed to density stratification effects along the coronal loop. Several examples have been published where the $P_{1} / P_{2}$ ratio is within the interval 1.5-1.9 (McEwan et al. 2006; Van Doorsselaere et al. 2007; Ruderman et al. 2008; Verth \& Erdélyi 2008; Verth et al. 2008). Andries et al. (2005) show how a one-to-one relationship exists between the $P_{1} / P_{2}$ ratio and the density scale-height. The dependence of the period ratio on $L / \pi H$, where $H$ is the density scale-height, is shown in Fig. 4.

According to Andries et al. (2005), the dependence of the period ratio on the density stratification does not depend on other loop parameters or the magnetic field strength. As a result, the same dependence can be demonstrated for a variety of density profiles generated inside the same coronal loop. For the coronal loop under current investigation, we find that $P_{1} / P_{2}=1.82 \pm 0.02$, corresponding to a density scale height

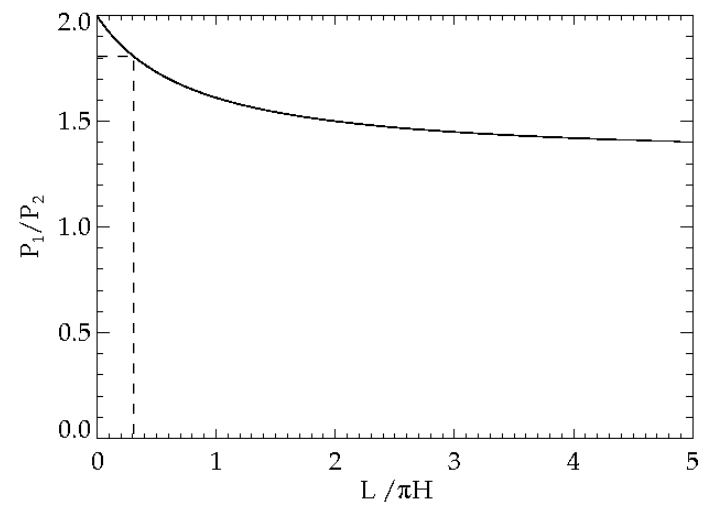

Fig. 4. The period ratio, $P_{1} / P_{2}$, corresponding to the fundamental to first harmonic kink mode frequencies, plotted as a function of loop length, $L$, and density scale height, $H$. A $P_{1} / P_{2}$ ratio equal to 1.82 , combined with a loop length $L \approx 73 \mathrm{Mm}$, provides a density scale height of $H \approx 72 \mathrm{Mm}$

$H=73 \pm 3 \mathrm{Mm}$. This value is consistent with those found by Van Doorsselaere et al. (2007).

Assuming that the loop is in hydrostatic equilibrium, the density scale height is directly proportional to the temperature, $T$, according to the equation

$$
H=47\left(\frac{T}{\mathrm{MK}}\right),(\mathrm{Mm}) \text {. }
$$

Thus, for our present analysis we obtain a loop temperature of $T=1.5 \pm 0.6 \mathrm{MK}$, which is within TRACE's temperature response for the $171 \AA$ channel, and towards the central portion of the $195 \AA$ channel's temperature response curve (Aschwanden 2004; Phillips et al. 2005). Nevertheless, the visibility of the coronal loop in both the $171 \AA$ and $195 \AA$ bandpasses, in addition to a derived loop temperature within the TRACE instrument's temperature response function, suggests that the loop under investigation is in hydrostatic equilibrium (Andries et al. 2005).

On the basis of the period of the fundamental mode (501 s), following the methods of Verwichte et al. (2004), it is possible to calculate the kink speed, $c_{\mathrm{K}}$, of the wave to be $c_{\mathrm{K}}=$ $2 L / P_{\text {fundamental }}=291 \pm 8.2 \mathrm{~km} \mathrm{~s}^{-1}$. The kink speed can then be related to the magnetic field strength, $B$, by the equation

$B=c_{\mathrm{K}}\left[\frac{\mu\left(\rho_{\mathrm{i}}+\rho_{\mathrm{e}}\right)}{2}\right]^{1 / 2}$,

where $\rho_{\mathrm{i}}$ and $\rho_{\mathrm{e}}$ are the internal and external loop densities, respectively, $\mu$ is the permeability of free space, and the magnetic field strength inside and outside the loop is assumed to be identical. Thus, we estimate the magnetic field strength inside the coronal loop to be $2.0 \pm 0.7 \mathrm{G}$, which is towards the lower-end of a statistical study compiled by Nakariakov \& Ofman (2001) and Aschwanden et al. (2002).

\subsection{Comparison with other theories}

The existence of two distinct periods in the loop under investigation has been explained and investigated assuming that the two periods belong to the fundamental and first harmonic kink oscillations of the loop. However, other viable explanations are also possible. For our discussion, we first recall the results of Ballai et al. (2008). Using a simple coronal loop model, these authors investigated the interaction between an EIT wave and a coronal loop assuming an equilibrium of forces. Their results 
show that the periods recovered in a coronal loop are always a combination of the period of the driver (here EIT waves) and the natural period of the loop. The same authors showed that the displacement of a coronal loop (here denoted by $Q(z, t))$ under the influence of a driver, $F(z, t)$, is given by

$\frac{\partial^{2} Q}{\partial t^{2}}-c_{\mathrm{K}}^{2} \frac{\partial^{2} Q}{\partial z^{2}}+\omega_{\mathrm{c}}^{2} Q=F$

where, as before, $c_{\mathrm{K}}$ is the kink speed (propagation speed of disturbances in the loop) and $\omega_{\mathrm{c}}$ is the cut-off frequency of kinkmode oscillations. These quantities are simply given by

$c_{\mathrm{K}}=\sqrt{\frac{\rho_{\mathrm{i}} v_{\mathrm{Ai}}^{2}+\rho_{\mathrm{e}} v_{\mathrm{Ae}}^{2}}{\rho_{\mathrm{i}}+\rho_{\mathrm{e}}}}, \quad \omega_{\mathrm{c}}=\frac{g\left(\rho_{\mathrm{i}}-\rho_{\mathrm{e}}\right)}{2 c_{\mathrm{K}}\left(\rho_{\mathrm{i}}+\rho_{\mathrm{e}}\right)}$,

where $v_{\mathrm{Ai}}$ and $v_{\mathrm{Ae}}$ are the Alfvén speeds inside and outside the loop. Equation (4) is an inhomogeneous Klein-Gordon equation that was solved assuming that the foot-points of the loop are fixed (line-tying condition) and initially at rest.

The event occurring on 13 June 1998 was also studied in detail by Wills-Davey \& Thompson (1999), who showed that the blast wave intersects the loop of interest nearly perpendicularly, meaning we can model the EIT wave as a harmonic driver whose temporal and spatial dependence is given by Ballai et al. (2008) to be

$F(z, t) \propto K\left[\delta\left(z-z_{0}\right)-\delta\left(z-L+z_{0}\right)\right] \mathrm{e}^{\mathrm{i} \omega_{\text {EIT }} t}$,

where $\delta(z)$ is the Dirac-delta function, and the constant $K$ depends on the density of the plasma and energy of the EIT wave, both considered to be constant. In Eq. (5), $\omega_{\text {EIT }}$ is the frequency of the EIT wave, which is assumed to be a harmonic signal. In this study, we are concerned with temporal changes only, which is why the exact form of the driver is not explicitly given. The form of the driver given by Eq. (5) reveals that the interaction between the loop and the EIT wave occurs in two points (at $z=z_{0}$ and $\left.z=L-z_{0}\right)$ simultaneously, and is placed symmetrically with respect to the end-points of the loop at $z=0$ and $z=L$.

Equation (4), where $F(z, t)$ is defined by Eq. (5), was solved by Ballai et al. (2008), with the temporal dependence of the displacement, $Q(z, t)$, found to be

$Q(z, t) \propto \sum_{n=1}^{\infty} \sin \left[\left(\frac{\omega_{n}+\omega_{\mathrm{EIT}}}{2}\right) t\right] \sin \left[\left(\frac{\omega_{n}-\omega_{\mathrm{EIT}}}{2}\right) t\right]$,

where $\omega_{n}^{2}=\omega_{\mathrm{c}}^{2}+n^{2} \pi^{2} c_{\mathrm{K}}^{2} / L^{2}(n \geq 1)$ are the natural frequencies of the loop.

According to the results of Ballai et al. (2008, their Fig. 3), the dominant (and strongest) signal in the loop is produced by the driver. Bearing in mind the previously presented model, we identify the two measured periods as the two temporal dependencies of the solution given by Eq. (6). Since it is visually obvious that the loop oscillates at its fundamental frequency, $\omega_{n}$ in Eq. (6), it is replaced by $\omega_{1}$ corresponding to the frequency of the fundamental mode. That means that we need to solve the system

$\omega_{1}+\omega_{\mathrm{EIT}}=\frac{4 \pi}{T_{2}}, \quad \omega_{1}-\omega_{\mathrm{EIT}}=\frac{4 \pi}{T_{1}}$,

where $T_{1}=501 \mathrm{~s}$ and $T_{2}=274 \mathrm{~s}$. It is easy to show that the period of the fundamental mode is $177.1 \pm 3.5 \mathrm{~s}$ and the period of the driving EIT wave is $604.7 \pm 26.8 \mathrm{~s}$, much higher than the period derived by Ballai et al. (2005). However, we should keep in mind that the result derived by Ballai et al. (2005) refers to an average period derived from within a large field-of-view.

Once the period of the fundamental mode is derived, it is straightforward to estimate the magnetic field strength inside the loop. We, again, need to use the mathematical finding of Ballai et al. (2008) to derive the magnetic field. According to their analysis, the frequency of the fundamental mode is given by

$\omega_{1}=\sqrt{\omega_{\mathrm{c}}^{2}+\pi^{2} c_{\mathrm{K}}^{2} / L^{2}}$.

Using the previously determined values for densities and length of the loop, together with the derived frequency of the fundamental mode, we obtain the fundamental kink speed of the loop to be $823 \pm 15 \mathrm{~km} \mathrm{~s}^{-1}$. Using the standard definition of the kink speed, and assuming the same magnetic field strength inside and outside the loop, we can obtain the magnetic field in the loop to be

$B=c_{\mathrm{K}} \sqrt{\frac{\mu\left(\rho_{\mathrm{i}}+\rho_{\mathrm{e}}\right)}{2}}=5.8 \pm 1.8 \mathrm{G}$.

The values of the magnetic field strength determined using two different scenarios are rather different, meaning that the determination of this fundamental quantity depends on the applied theoretical model used to explain the observed periods.

As we specified earlier the interpretation of two periods observed in a loop is not unique because different scenarios can occur. Unfortunately, given the present spatial resolution constraints, it is impossible to distinguish between these models, meaning that all findings derived from the observations of loop oscillations should be treated with care.

Another possible explanation of the two observed periods is connected to the limits of the spatial resolution of the TRACE instrument. The two observed periods could belong to two neighboring thin loops that cannot be resolved by our observations moving in phase. This possibility was studied using numerical simulations by Luna et al. (2008), and using theoretical models by Van Doorsselaere et al. (2008), Robertson et al. (2010), and Robertson \& Ruderman (2011). Assuming that the loops are identical, we can easily find that the ratio of $d / R$, where $d$ is the distance between the longitudinal axes of the two loops and $R$ is their radius, is $2 \pm 0.11$. Thus, the physical distance between the two loops, in units of their radius, is $0.031 \pm 0.001$. Furthermore, using the results of Van Doorsselaere et al. (2008), we can estimate the magnetic field inside the loop to be $2.6 \pm 0.4 \mathrm{G}$, a value remarkably consistent with that obtained when interpreting the two periods as belonging to the fundamental and first harmonic kink mode of a single coronal loop.

Finally, the fourth possible explanation is related to the intrinsic properties of the coronal loop where the second harmonic is not a proper eigenmode but just reflects a slightly more triangular amplitude profile than sinusoidal. In other words, the second period is observed as some nonlinear amplitude oscillation caused by a gradient in either density or magnetic field across the loop motion, or a coupling with neighboured loops.

\section{Conclusions}

To carry out coronal seismology it is imperative to know the relationship between the composition of a plasma structure and the oscillations supported by the coronal loop. High resolution observations enable us to accurately measure not only the magnetic field strength, but also the thermodynamical state of the plasma. 
The event studied here occurred on 13 June 1998, and was recorded by the TRACE instrument in both $171 \AA$ and $195 \AA$ bandpass filters. A sudden energy release outside the field-ofview generated a global wave that interacted with local coronal loops. The resulting loop oscillations were identified as kink modes, i.e. oscillations that propagate along the coronal loop leading to the displacement of the longitudinal symmetry axis of the tube. Rigorous analysis of the oscillation revealed the existence of two distinct periods contained within a relatively short loop. Periodicities of $501 \pm 5 \mathrm{~s}$ and $274 \pm 7 \mathrm{~s}$ allowed us to draw conclusions about the magnetic field strength and the longitudinal density structure inside the loop. The magnetic field established here has a value towards the lower end of recent statistical studies. However, a $30 \%$ increase in the magnetic field strength can be achieved if the loop filling factor is doubled. A major conclusion of our study is that two observed periodicities in a loop structure might not be a unique interpretation, often with derived system parameters differing substantially owing to the independent seismological methods. We have compared the observational evidence with two different theoretical interpretations of oscillations. First, we considered that the two periods are the periods corresponding to the fundamental and first harmonic. The $P_{1} / P_{2}=1.82 \pm 0.02$ ratio of the two periods has allowed us to determine values of $73 \pm 3 \mathrm{Mm}$ and $1.5 \pm 0.6 \mathrm{MK}$ for the density scale height and loop temperature, respectively. Furthermore, an estimate of the magnetic field strength inside the coronal loop was determined to be $2.0 \pm 0.7 \mathrm{G}$. All values determined are consistent with previous studies involving coronal seismology, even though the coronal loop structure presented here is relatively confined and dim.

A second possible explanation of two periods might be that developed by Ballai et al. (2008), which states that the two periods might belong to the driver (here an incident EIT wave assumed to be harmonic) and the visibly clear fundamental mode kink oscillation. Using the results of Ballai et al. (2008), we obtained the period of the independent driver and kink oscillation, with the latter quantity allowing us to again estimate the magnetic field strength inside the loop structure. The theory developed by Ballai et al. (2008) relies heavily on the EIT wave being described using wave theory, as opposed to a propagating magnetic feature. Our observations provide additional support that wave theory can describe the mechanism behind EIT waves. As such, we feel the use of EIT waves for coronal seismology is an accurate and reliable approach. The magnetic field determined in light of this scenario is almost triple that obtained utilising the first method. On the basis of the magnetic field strength alone, and its consistency with previous estimated values, we deem that the interpretation of the two periods as the combination of the loop's natural fundamental period and the period of the harmonic driver, seems more plausible. Furthermore, when the two periods were assumed to belong to two unresolved coronal threads we were able to estimate both the magnetic field inside the threads, in addition to the distance between them. Finally, another possible explanation of the two periods was linked to the significant density or magnetic field gradient along the motion path of the oscillating loop, or an aspect of coupling with neighbouring loop structures.

Our study reveals that the periods of oscillations of a coronal loop should be interpreted with great care as a multitude of scenarios are plausible that would each result in different values of, e.g. magnetic field. Further higher resolution observational evidence will help us to apply the most likely interpretation of dynamical processes.

Acknowledgements. I.B. acknowledges the financial support by NFS Hungary (OTKA, K83133). D.B.J. wishes to thank the Science and Technology Facilities Council for the award of a Post-Doctoral Fellowship. M.D. acknowledges the support of STFC. We are grateful to the anonymous referee for his/her comments and for pointing out the fourth possible explanation of two periods in a coronal loop.

\section{References}

Andries, J., Arregui, I., \& Goossens, M. 2005, ApJ, 624, L57

Andries, J., van Doorsselaere, T., Roberts, B., et al. 2009, Space Sci. Rev., 149,

Aschwanden, M. J. 2004, Physics of the Solar Corona (Berlin: Springer-Verlag) Aschwanden, M. J., Tarbell, T. D., Nightingale, R. W., et al. 2000, ApJ, 535, 1047

Aschwanden, M. J., de Pontieu, B., Schrijver, C. J., \& Title, A. M. 2002, Sol. Phys., 206, 99

Ballai, I. 2007, Sol. Phys., 246, 177

Ballai, I., Erdélyi, R., \& Pintér, B. 2005, ApJ, 633, L145

Ballai, I., Douglas, M., \& Marcu, A. 2008, A\&A, 488, 1125

Banerjee, D., Erdélyi, R., Oliver, R., \& O'Shea, E. 2007, Sol. Phys., 246, 3

Christian, D. J., Drake, J. J., \& Mathioudakis, M. 1998, AJ, 115, 316

De Moortel, I., \& Brady, C. S. 2007, ApJ, 664, 1210

Delannée, C. 2000, ApJ, 545, 512

Edwin, P. M., \& Roberts, B. 1983, Sol. Phys., 88, 179

Fludra, A., Del Zanna, G., Alexander, D., \& Bromage, B. J. I. 1999, J. Geophys. Res., 104, 9709

Golub, L., Bookbinder, J., Deluca, E., et al. 1999, Phys. Plasmas, 6, 2205

Jess, D. B., Andić, A., Mathioudakis, M., Bloomfield, D. S., \& Keenan, F. P. 2007a, A\&A, 473, 943

Jess, D. B., McAteer, R. T. J., Mathioudakis, M., et al. 2007b, A\&A, 476, 971

Jess, D. B., Mathioudakis, M., Erdélyi, R., et al. 2008, ApJ, 680, 1523

Lin, A. C., Nightingale, R. W., \& Tarbell, T. D. 2001, Sol. Phys., 198, 385

Luna, M., Terradas, J., Oliver, R., \& Ballester, J. L. 2008, ApJ, 676, 717

McEwan, M. P., Donnelly, G. R., Díaz, A. J., \& Roberts, B. 2006, A\&A, 460, 893

Nakariakov, V. M., \& Ofman, L. 2001, A\&A, 372, L53

Nakariakov, V. M., Ofman, L., Deluca, E. E., Roberts, B., \& Davila, J. M. 1999, Science, 285,862

O’Shea, E., Srivastava, A. K., Doyle, J. G., \& Banerjee, D. 2007, A\&A, 473, L13

Phillips, K. J. H., Chifor, C., \& Landi, E. 2005, ApJ, 626, 1110

Press, W. H., Teukolsky, S. A., Vetterling, W. T., \& Flannery, B. P. 1992, 2nd edn. (Cambridge: University Press)

Roberts, B., Edwin, P. M., \& Benz, A. O. 1984, ApJ, 279, 857

Robertson, D., \& Ruderman, M. S. 2011, A\&A, 525, A4

Robertson, D., Ruderman, M. S., \& Taroyan, Y. 2010, A\&A, 515, A33

Ruderman, M. S., \& Erdélyi, R. 2009, Space Sci. Rev., 149, 199

Ruderman, M. S., Verth, G., \& Erdélyi, R. 2008, ApJ, 686, 694

Selwa, M., Murawski, K., Solanki, S. K., \& Ofman, L. 2010, A\&A, 512, A76

Ugarte-Urra, I., Doyle, J. G., \& Madjarska, M. S. 2002, SOLMAG 2002, Proceedings of the Magnetic Coupling of the Solar Atmosphere Euroconference, 505, 595

Van Doorsselaere, T., Nakariakov, V. M., \& Verwichte, E. 2007, A\&A, 473, 959 Van Doorsselaere, T., Ruderman, M. S., \& Robertson, D. 2008, A\&A, 485, 849 Verth, G., \& Erdélyi, R. 2008, A\&A, 486, 1015

Verth, G., Van Doorsselaere, T., Erdélyi, R., \& Goossens, M. 2007, A\&A, 475, 341

Verth, G., Erdélyi, R., \& Jess, D. B. 2008, ApJ, 687, L45

Verwichte, E., Nakariakov, V. M., Ofman, L., \& Deluca, E. E. 2004, Sol. Phys., 223, 77

Wills-Davey, M. J., \& Thompson, B. J. 1999, Sol. Phys., 190, 467 